This article was published in Journal of Biomaterials Application, 28(9), 1325-1335, 2014 http://dx.doi.org/10.1177/0885328213507300

\title{
Influence of nanohydroxyapatite surface properties on Staphylococcus epidermidis biofilm formation
}

\author{
J Barros ${ }^{1,2,4}$, L Grenho ${ }^{1,2}$, CM Manuel ${ }^{4,5}$, C Ferreira ${ }^{4}$, L Melo ${ }^{4}$, \\ OC Nunes ${ }^{4}$, FJ Monteiro ${ }^{1,2}$ and MP Ferraz ${ }^{1,3}$ \\ 'INEB - Instituto de Engenharia Biomédica, Universidade do Porto, Porto, Portugal \\ ${ }^{2}$ Departamento de Engenharia Metalúrgica e Materiais, FEUP - Faculdade \\ de Engenharia - Universidade do Porto, Porto, Portugal \\ ${ }^{3}$ CEBIMED - Centro de Estudos em Biomedicina, Universidade Fernando Pessoa, Porto, Portugal \\ ${ }^{4}$ LEPAE - Laboratório de Engenharia dos Processos, Ambiente e Energia, \\ Departamento de Engenharia Qu'ı́mica, Faculdade de Engenharia - Universidade do Porto, Porto, \\ Portugal \\ ${ }^{5}$ ULP - Universidade Lusófona do Porto, Porto, Portugal \\ Corresponding author: \\ Joana Barros, INEB - Instituto de Engenharia Biomédica, Universidade do \\ Porto, Rua do Campo Alegre, 823 Porto, 4150-180, Portugal. Email: joanabarros@fe.up.pt
}

\begin{abstract}
Nanohydroxyapatite (nanoHA), due to its chemical properties, has appeared as an exceptionally promising bioceramic to be used as bone regeneration material. Staphylococcus epidermidis have emerged as major nosocomial pathogens associated with infections of implanted medical devices. In this work, the purpose was to study the influence of the nanoHA surface characteristics on $S$. epidermidis RP62A biofilm formation. Therefore, two different initial inoculum concentrations $\left(\mathrm{C}_{\mathrm{i}}\right)$ were used in order to check if these would affect the biofilm formed on the nanoHA surfaces. Biofilm formation was followed by the enumeration of cultivable cells and by scanning electron microscopy. Surface topography, contact angle, total surface area and porosimetry of the biomaterials were studied and correlated with the biofilm data. The surface of nanoHA sintered at $830^{\circ} \mathrm{C}$ (nanoHA830) showed to be more resistant to S. epidermidis attachment and accumulation than that of nanoHA sintered at $1000^{\circ} \mathrm{C}$ (nanoHAl000). The biofilm formed on nanoHA830 presented differences in terms of structure, surface coverage and EPS production when compared to the one formed on nanoHAI000 surface. It was observed that topography and surface area of nanoHA surfaces had influence on the bacterial attachment and accumulation. $\mathrm{C}_{i}$ influenced bacteria attachment and accumulation on nanoHA surfaces over time. The choice of the initial inoculum concentration was relevant proving to have an effect on the extent of adherence thus being a critical point for human health if these materials are used in implantable devices. This study showed that the initial inoculum concentration and surface material properties determine the rate of microbial attachment to substrata and consequently are related to biofilm-associated infections in biomaterials.
\end{abstract}

Keywords

Inoculum concentration, biofilm formation, nanohydroxyapatite, biomaterial roughness, biomaterial surface area 


\section{Introduction}

Nanoscale-engineered surfaces have been applied in biomedical implants and prosthetic devices in order to modulate, understand and control the interactions between biomaterials and biological systems. ${ }^{1}$ Nanohydroxyapatite (nanoHA), and generally bio- active calcium phosphate materials, have received considerable attention as materials for implants and bone augmentation procedures, because they chemically bind directly onto bone when implanted, resulting in the formation of a strong bone/implant interface. ${ }^{2}$ NanoHA possesses exceptional biocompatibility and bioactivity with respect to bone cells and tissues, perhaps due to its similarity with the mineral component of hard tissues. Also, nanoHA promotes ion exchange with the physiological environment, and increases protein adsorption and cellular response. ${ }^{3,4}$ Due to these properties, nanoHA has been used as a bulk material or as a coating on orthopedic and dental implants. ${ }^{5}$

Additionally, the capacity of nanoHA to be a vehicle for the transport of biochemical factors or drugs, respectively, for tissue engineering or cancer treatment has been assessed. ${ }^{6,7}$

Bacterial infections associated to prosthetic implants and medical devices present strong clinical challenges. The micro-organisms are able to attach to biomaterials used in prosthetic implants and medical devices, consequently causing biomaterials-related infections in humans. ${ }^{8}$ The colonization and infection of orthopaedic implants depend on many factors, including the biomaterial surface topography and roughness and surface chemistry, environmental factors and characteristics of bacterial cells. Staphylococcus epidermidis has emerged as a major nosocomial pathogen associated to implanted medical devices. ${ }^{9,10}$ The ability of

S. epidermidis as a pathogen in hospital environment can be explained by its highly adaptative nature, inherent genetic variability and intrinsic genetic flexibility, ${ }^{11}$ all of which enable it to withstand hostile external environments. ${ }^{9}$ This organism, which is among the most prevalent bacteria in human skin and mucous microbiota, presents unique problems of diagnosis and infection treatment involving biofilm formation on implanted biomaterials. ${ }^{10}$ The ability to adhere to biomaterials and form a stable biofilm is considered to be an important virulence factor of $S$. epidermidis. ${ }^{12}$ An extracellular polysaccharide adhesin (PIA), encoded by the icaADBC locus, represents a key virulence determinant in $S$. epidermidis and is required for biofilm formation. ${ }^{12}$

Biofilms form on inert or living surfaces and are composed of heterogeneous communities of bacteria functionally organized and enclosed in a self-produced polymeric matrix, named extracellular polysaccharide substance (EPS). Generally biofilm development may be composed by three steps: the lag phase, a conditioning phase during which bacteria attach and colonize the surface; the growth phase corresponding to exponential accumulation under quasi-steady state. ${ }^{13}$ Biofilm development processes are influenced by multiple factors such as temperature, $\mathrm{pH}$, nutrients availability, material surface, bacteria type and concentration. ${ }^{13,14}$ Wang et al. ${ }^{15}$ showed that low levels of adherent bacteria can be enough to potentiate an infection. For $S$. aureus, $4 \times 10^{3}$ to $4 \times 10^{7} \mathrm{CFU} / \mathrm{mL}$ are required to develop infections in rabbit models after orthopedic implants. ${ }^{8}$ The initial inoculum concentration can influence the bacterial adhesion and biofilm formation on ceramic implants. In this work, the purpose was to study the influence of the characteristics of nanoHA surfaces, resulting from two different sintering heat-treatments, on S. epidermidis RP62A biofilm formation. Therefore, two different initial inoculum concentrations $\left(\mathrm{C}_{\mathrm{i}}\right)$ were used in order to check if these $\mathrm{C}_{\mathrm{i}}$ would affect the biofilm formed on the nanoHA surfaces. Biofilm formation was followed by cultivable cell numbers (CFUs) and the biofilm structure was visualized by scanning electron microscopy (SEM). The biomaterial surface was 
characterized in terms of surface topography, con- tact angle, total surface area and porosimetry and correlated with biofilm data.

\section{Materials and methods \\ Preparation of nanohydroxyapatite materials}

Powders of nanohydroxyapatite (nanoHA) were kindly supplied by Fluidinova S.A., Portugal (nanoXIM_HAp202). Cylindrical nanoHA discs were obtained using $150 \mathrm{mg}$ of dry powder under uniaxial compression stress of $8 \mathrm{MPa}$ (Mestra Snow P3). Two different sintering temperatures were used, namely $830^{\circ} \mathrm{C}$ (nanoHA830) and $1000^{\circ} \mathrm{C}$ (nanoHA1000), with a $15 \mathrm{~min}$ plateau and applying a heating rate of $20^{\circ} \mathrm{C} / \mathrm{min}$. The sintering cycle was completed with a natural cooling process inside the furnace. All the experimental conditions related to the compression and sintering procedure previously referred were optimized in order to have stable discs maintaining a nanoscale grain sizes after heat-treatment. The choice of a heat-treatment cycle was done based on the assumption that these materials require sintering to acquire the adequate mechanical strength to withstand manipulation during the experiments. NanoHA ceramics are very brittle materials if not adequately heat-treated, as are most ceramic materials. Our choices of temperatures were done so that we would be closely above the optimal sintering temperature of nanoHA samples, and we have selected just $30^{\circ}$ above the optimal sintering temperature $\left(800^{\circ} \mathrm{C}\right.$, obtained from scanning differential calorimetry measurements) to ensure that all parts of the samples had reached that temperature inside the furnace, and another one slightly above $\left(1000^{\circ} \mathrm{C}\right)$ the theoretical sintering temperature to minimize the effect of sintering on crystal morphology and crystal growth and therefore keep the nanostructure. The samples were sterilized by two passages with ethanol 70\% during 15 min followed by a double washing in sterile $0.9 \% \mathrm{NaCl}$.

\section{Material characterization}

The linear expansion behavior of "green", i.e. prior to sintering heat-treatment, compacted nanoHA discs, during sintering was studied using a high-resolution dilatometer (Setsys $16 / 18$, Setaram, France), at a heating rate of $5^{\circ} \mathrm{C} / \mathrm{min}$ from $100^{\circ} \mathrm{C}$ to $1400^{\circ} \mathrm{C}$.

Wettability studies were performed using Contact Angle measurements by the sessile drop technique using a Data Physics measurement system (model OCA 15, Germany). Due to the absorbing nature of nanoHA surfaces, the deposition of $4 \mathrm{~mL}$ of ultra-pure water drops on each material surface was recorded by a video charge-coupled device (CCD) camera and analyzed to obtain the contact angles. Values reported are the average of 10 measurements (one drop per each sample and side was randomly picked).

Zeta potential (ZP) was measured to evaluate the negative net charge of nanoHA surfaces, using an electrokinetic analyzer (EKA), applying the "automatic" mode method. ZP measurements were performed at pH 6 in $1 \mathrm{mM} \mathrm{KCl}$. Mean and standard deviation (SD) values of ZP of the different materials were calculated.

Mercury porosimetry method (Quantachrome Poremaster model No. 60) was used to assess the apparent density, total surface area, and pores volume percentage, pore size range of mesopores (pore diameter from 2 to $50 \mathrm{~nm}$ ) and macropores (pore diameter above $50 \mathrm{~nm}$ ) on different types of sintered discs (nanoHA830 and nanoHA1000). In this procedure, $0.4 \mathrm{~g}$ of each dried material was penetrated by mercury at increasingly high pressures and the reported data were obtained using Quantachrome Poremaster for Windows, versions 3.0 and 4.02. 
The morphologic characterization of the surfaces was obtained by SEM, using a FEI Quanta 400FEG/ ESEM microscope (FEI, USA). NanoHA1000 and nanoHA830 discs were sputtercoated with a thin gold/palladium film, using a sputter coater (SPI- Module) in an argon atmosphere before analysis. Five fields for each sample were randomly chosen, under a 50,000 magnification.

Surface topography was examined by atomic force microscopy (AFM) under "tapping mode" using a NanoSeøpe Iva (Veeco Multimode, Digital Instruments, Santa Barbara, CA) equipped with silicon nitride tips (Veeco RTESP, Digital Instruments, Santa Barbara, CA). Each sample was imaged with of $10 \times 10 \mu \mathrm{m}^{2}$ scanned area. The surfaces were analyzed by measuring the average surface roughness (Ra) of 10 randomly chosen images per sample from selected areas of $2 \times 2 \mu \mathrm{m}^{2}$. Three replicas were used. The results referred to Ra correspond to the mean $\pm \mathrm{SD}$. Ra is defined as the average absolute deviation of the roughness irregularities from the mean line over one sampling length and gives a good gen?eral description of height variations. ${ }^{16}$

\section{Bacterial strain and broth culture preparation}

Tryptic Soy Broth (TSB) and Plate Count Agar (PCA) (Liofilchem, Italy) were prepared according to the manufacturer's instructions. Physiological saline was prepared adding $0.9 \% \mathrm{NaCl}$ (J.T.Baker, Denmark) to distilled water. S. epidermidis strain RP62A (ATCC 35984), a slime producer, ${ }^{12}$ was used in all experiments hereby reported. The test strain was incubated in $15 \mathrm{~mL}$ of TSB from a PCA culture not older than two days, for $24( \pm 2) \mathrm{h}$ at $37^{\circ} \mathrm{C}$ and $150 \mathrm{rpm}$, in an orbital shaker (Certomat ${ }^{\circledR} \mathrm{HK}, \mathrm{B}$. Braun Biotech International, Goettingen). Then, $50 \mathrm{~mL}$ were transferred to $150 \mathrm{~mL}$ of fresh TSB, and ållowed to grow for $18( \pm 2) \mathrm{h}$, at $37^{\circ} \mathrm{C}$ and $150 \mathrm{rpm}$. Bacterial cells were harvested by centrifugation (Ependorf $5804^{\circledR}$, Germany) for $10 \mathrm{~min}$ at $4800 \mathrm{rpm}$ and $4^{\circ} \mathrm{C}$. The bacterial cells were re-suspended in physiological saline solution in order to obtain two different densities: $1.23 \times 10^{8}$ cells $/ \mathrm{mL}$ $\left(\mathrm{OD}_{640} \mathrm{~nm}=0.15\right)$ and $1.23 \times 10^{5}$ cells $/ \mathrm{mL}$. The last one was obtained from serial dilutions from the higher density.

\section{Biofilm formation on nanoHA discs}

S. epidermidis RP62A biofilm formation was assessed on nanoHA discs, that were used as bacterial substrates in this assay. The sterilized samples were introduced on test tubes containing $2 \mathrm{~mL}$ TSB. Then $8 \mathrm{~mL}$ of previ- ously prepared bacterial suspensions was added and incubated at $37^{\circ} \mathrm{C}$ and $150 \mathrm{rpm}$ for 24,48 and $72 \mathrm{~h}$. Every $24 \mathrm{~h}$, the medium was carefully replaced by fresh TSB. The discs were gently washed with $0.9 \% \mathrm{NaCl}$, immersed in a flask containing $25 \mathrm{~mL}$ of sterile saline and sonicated for $10 \mathrm{~min}$ in an ultra-sonic bath (Transsonic 420 ELMA, $70 \mathrm{~W}, 35 \mathrm{kHz}$ ) to release the attached bacteria into the suspension. Negative controls were obtained by incubating discs in TSB without adding any bacterial cells. All experiments were performed in triplicate.

\section{Cultivable cell numbers}

The heterotrophic plate count is a procedure for estimating the number of colony-forming units (CFU), which corresponds to the cultivable bacteria. The agar medium used was PCA. The cultivable bacteria counts of the diluted biofilm suspensions were estimated by the spread plating method using $100 \mu \mathrm{L}$ of the dilutions from dispersed biofilm. The plates were incubated for $24 \mathrm{~h}$ at $37^{\circ} \mathrm{C}$. The counted plates contained between 30 and $300 \mathrm{CFUs}$. 
The average and the standard deviation of the biofilm density samples were adjusted to the disc area.

\section{Scanning electron microscopy}

After biofilm formation, specimen of each material and for each time (only for $1.23 \times 10^{8}$ cells / mL initial concentration) was fixed in $3 \%(\mathrm{v} / \mathrm{v}$ ) glutaraldehyde (Flukx Germany), for $20 \mathrm{~min}$ at room temperature and subsequently dehydrated by several steps using ethanolwater solutions, for $10 \mathrm{~min}$ each, with increasing concentrations of ethanol up to $100 \%$. The samples in absolute ethanol were taken to critical point drier, using $\mathrm{CO}_{2}(\mathrm{CPD}$ 7501, Polaron Range). The samples were then sputter-coated (SPI-Module) with a thin gold/palladium film and analyzed by SEM using an FEI Quanta 400FEG/ESEM microscope (FEI, USA) (Accelerating Voltage: $15 \mathrm{kV}$ ). For each sample five fields were randomly chosen to eliminate possible uneven bacterial distribution, with magnifications between 1000 and 5000x. Whenever necessary, higher magnifications were used to assess the bacterial morphology and material surfaces or the interactions between them.

\section{Statistical analysis}

All the biofilm assays were compared using one-way analysis of variance (ANOVA), followed by post-hoc comparisons for all possible combinations of group means applying the Tukey HSD multiple comparison test using SPSSV ${ }^{\circledR}$ Statistics (vs. 19.0, Chicago). In all cases $p<0.05$ was chosen to denote the significance level.

\section{Results}

\section{Material characterization}

Figure 1 shows the linear expansion curve of compacted nanoHA discs starting from "green" (non-sintered) state throughout the sintering process. The graph shows that contraction started at ca. $800^{\circ} \mathrm{C}$ and it continued progressively up to $1300^{\circ} \mathrm{C}$ with the highest contraction reached at $950^{\circ} \mathrm{C}$ (double arrow). The first derivate curve exhibits three minimum points (triple arrows) at $800^{\circ} \mathrm{C}, 1000^{\circ} \mathrm{C}$ and $1100^{\circ} \mathrm{C}$. Maximal sintering contraction was $17 \%$.The reason for the choice of the sintering temperature for this material at $830^{\circ} \mathrm{C}$ has to do with the fact that it was desired to be within the sintering interval, but as closely as possible to the minimum temperature $\left(800^{\circ} \mathrm{C}\right)$ to avoid crystal growth and therefore keep the nanostructure, and an increase of $30^{\circ} \mathrm{C}$ was chosen to make sure that all the volume of each sample was above the minimum temperature during sintering cycles. Therefore, two different types of hydroxyapatite discs were prepared according to the procedure described in the previous section "Preparation of nano- hydroxyapatite materials": nanoHA sintered at $830^{\circ}$ and nanoHA sintered at $1000^{\circ} \mathrm{C}$.

No significant differences in the water contact angle between the nanoHA830 and nanoHA1000 surfaces were observed. Both materials showed to be hydro- philic with $\phi<90^{\circ}$ (Table 1$) .{ }^{17}$

The results of $\mathrm{ZP}$ showed that both surfaces were negatively charged; however, nanoHA1000 presented itself as the most negatively charged (Table 1).

Mercury porosimetry showed that nanoHA830 materials presented a bimodal pore distribution in the range of macropores and of large mesopores and a higher total porosimetry (35.2\%), and lower 


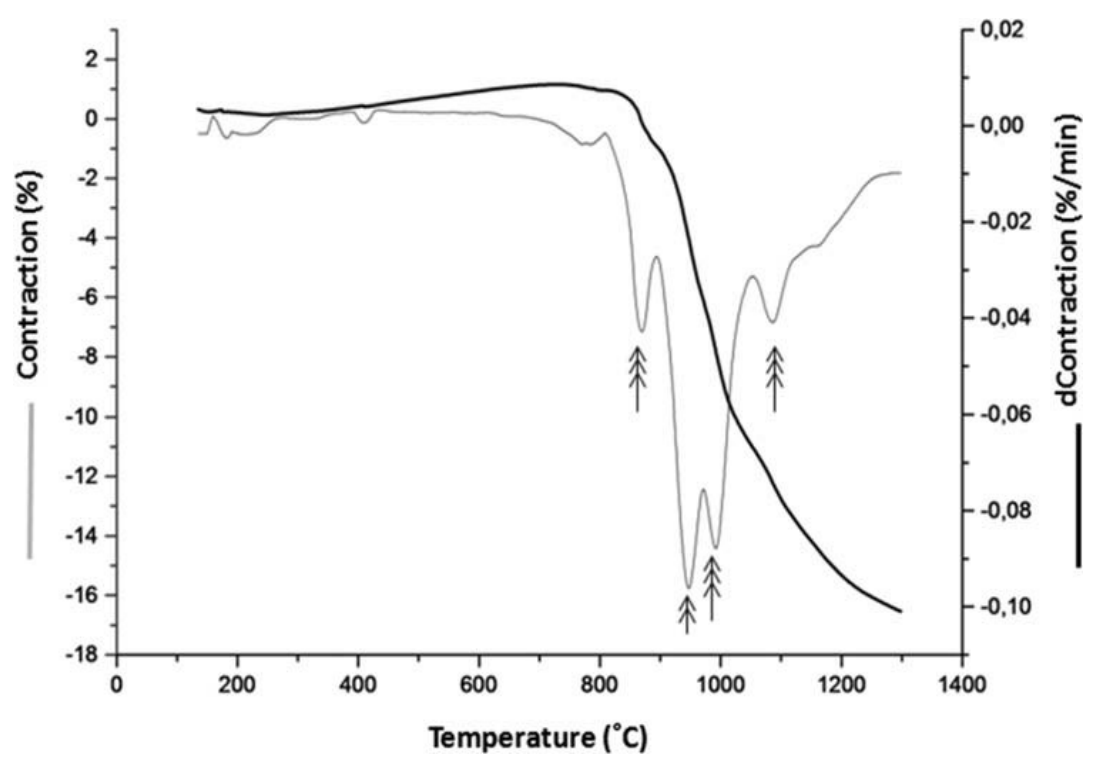

Figure 1. Linear expansion curve of "green" compacted nanoHA discs during sintering. Double arrow indicates highest contraction $\left(950^{\circ} \mathrm{C}\right)$ and triple arrows represent three minimum points $\left(800^{\circ} \mathrm{C}, 1000^{\circ} \mathrm{C}\right.$ and $\left.1100^{\circ} \mathrm{C}\right)$.

Table 1. Contact angle measurements, zeta potential, porosimetry parameters, surface area and roughness of the tested nanoHA biomaterials surfaces.

\begin{tabular}{lll}
\hline & \multicolumn{2}{l}{ Biomaterial surfaces } \\
\cline { 2 - 3 } & NanoHA830 & NanoHAI000 \\
\hline Contact angle $\left({ }^{\circ}\right)$ & $11.7 \pm 25^{\mathrm{a}}$ & $18.1 \pm 6.3^{\mathrm{a}}$ \\
Zeta potential $(\mathrm{mV})$ & $-5.9 \pm 0.1^{\mathrm{b}}$ & $-18.2 \pm 2.5^{\mathrm{a}}$ \\
Mean pore diameter $(\mathrm{m})$ & $6.0 \times 10^{-7 \mathrm{c}}$ & $9.8 \times 10^{-9}$ \\
& $3.9 \times 10^{-8}$ & \\
Total surface area $\left(\mathrm{m}^{2} / \mathrm{g}\right)$ & 4.9 & 9.8 \\
Theoretical total porosity $(\%)$ & 35.2 & 6.8 \\
Ra 2 $2 \mu \mathrm{m}^{2}(\mathrm{~nm})$ & $20.4 \pm 3.8^{\mathrm{a}}$ & $36.4 \pm 8.9^{\mathrm{b}}$ \\
\hline
\end{tabular}

2.b Significant differences $(p<0.05)$.

'Bi-modal distribution.

NanoHA: nanohydroxyapatite. 
surface area. Different results were obtained for the nanoHA1000 discs that presented a normal distribution in the mesopores range with an average diameter of approximately 10 $\mathrm{nm}$, and a lower total porosity (6.8\%), and higher surface area (Table 1).

Average Ra and surface topography were obtained by AFM studies over a $2 \times 2 \mu \mathrm{m}^{2}$ selected area. Concerning $\mathrm{Ra}$, siłnificant differences between nanoHA surfaces were observed. NanoHA830 surface exhibited lower Ra values than nanoHA1000 surface (Table 1). Regarding surface topography, AFM images of nanoHA surfaces (Figure 2) showed that nanoHA830 surface exhibit smaller grain size and larger porous diameters comparatively with nanoHA1000 surface. The results of roughness obtained from AFM may be quite misleading, although the values for Ra for nanoHA1000 might indicate the presence of higher peaks and deeper valleys, the frequency of topography chances, i.e. the number of rough peaks is much higher for the nanoHA830 samples, even if the difference between higher peaks and deeper valleys is smaller.

Figure 3 shows SEM micrographs of nanoHA830 and nanoHA1000 surfaces. It was observed, for both materials, that the nanoparticles associate into aggregates and the nano-dimensions of crystals were maintained. However, nanoHA1000 surface displays a more regular structure, with larger aggregates when compared to the nanoHA830 surface.

\section{Biofilm formation on nanohydroxyapatite surface over time}

In this work, the purpose was to study the influence of nanoHA surface characteristics on $S$. epidermidis RP62A biofilm formation. Therefore, two different ini- tial inoculum concentrations $\left(\mathrm{C}_{\mathrm{i}}\right)$ were observed, in order to check if these $\mathrm{C}_{\mathrm{i}}$ would affect the biofilm formed on the used nanoHA surfaces.

The results obtained showed different profiles of $S$. epidermidis attachment and accumulation on nanoHA830 and nanoHA1000 surfaces. For nanoHA1000 surface, an increase of bacteria accumulation was observed up to $48 \mathrm{~h}(p<0.05)$, followed by steadystate ( $p>0.05)$ (Figure 4(a), 4(b)). On the other hand, for nanoHA830 surface it was observed that the biofilm accumulation increased over time, noticing that up to $48 \mathrm{~h}$ it presented an initial lag conditioning phase, and at $72 \mathrm{~h}$ a high increase of the bacteria amount was observed (Figure 4(a) and (b)).

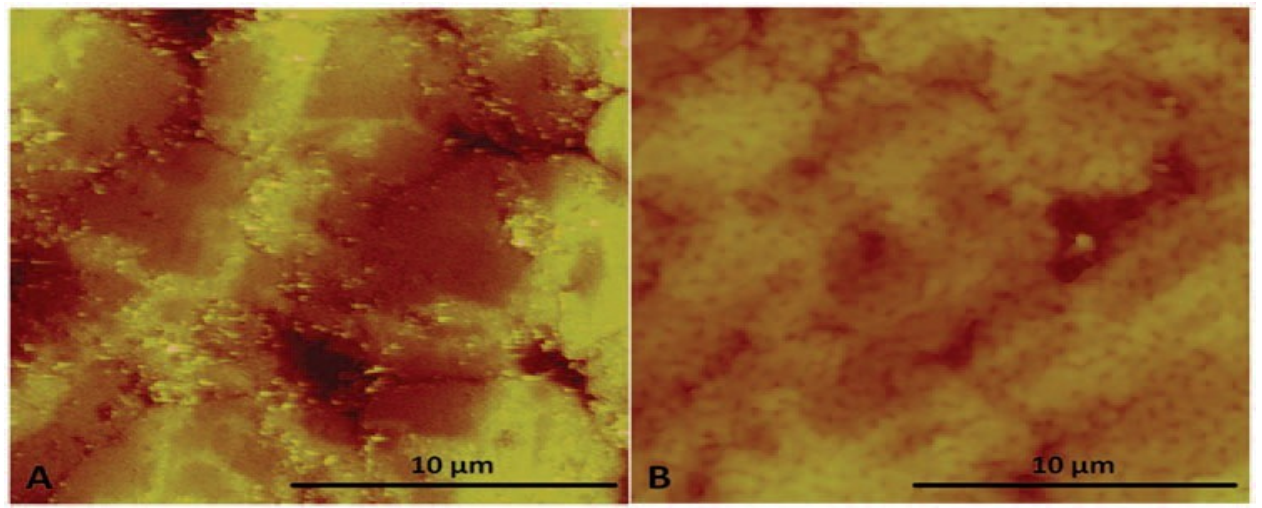

Figure 2. AFM images of nanoHA830 surface (a) and nanoHAI000 surface. (b) The images correspond to scanned areas of $10 \times 10 \mu \mathrm{m}^{2}$ each. 

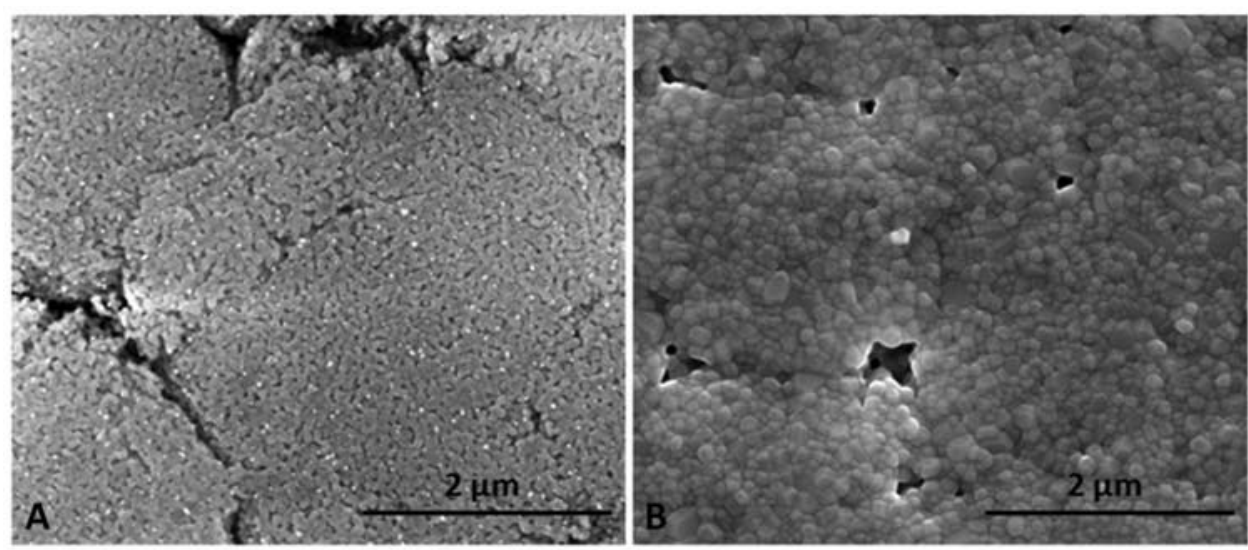

Figure 3. SEM images of nanoHA830 surface (a) and nanoHAI000 surface. (b) Area image was $2 \times 2 \mathrm{~mm}$ and 50,000x magnification.
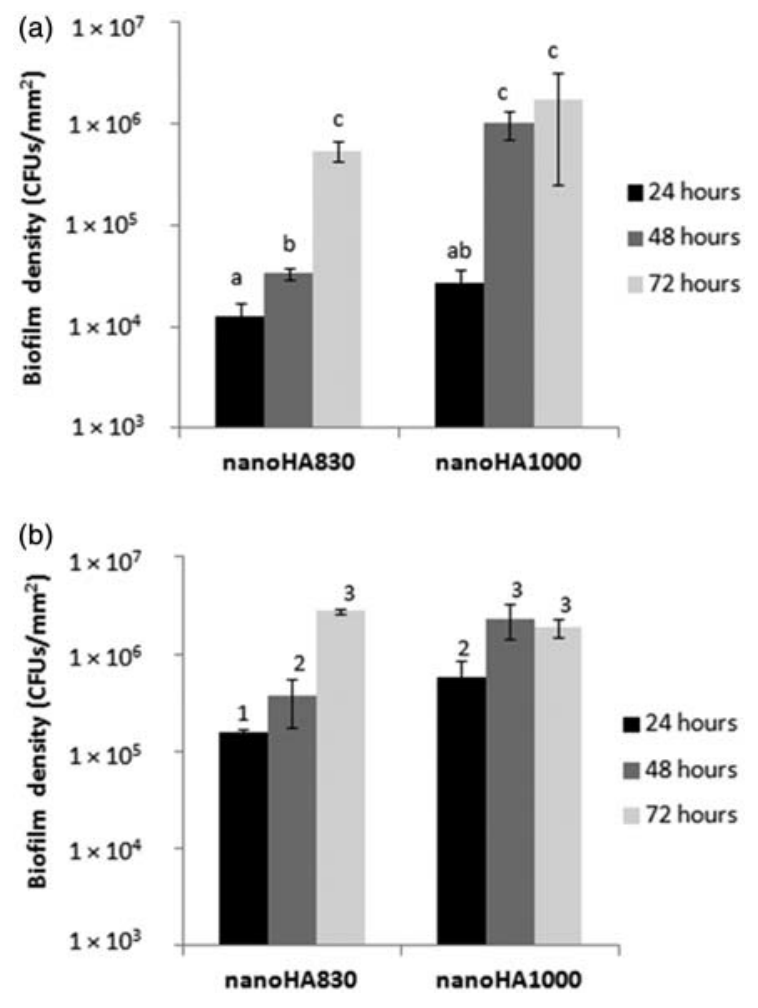

Figuire 4. Attached cells per unit surface area (cultivable cells numbers) for biofilms growth on nanoHA830 surface and nanoHAI000 surface in system fed-batch. (a) Biofilm density from $C_{i}=$ $1.23 \times 10^{5}$ cells $/ \mathrm{mL}$ (b) Biofilm density from $C_{i}=1.23 \times 10^{8}$ cells $/ \mathrm{mL}$. Different lowercase letters and numbers indicate significant differences ( $p$ 0.05) according to Tukey HSD.

For $\mathrm{Ci}=1.23 \times 10^{5}$ cells $/ \mathrm{mL}$, S. epidermidis showed, after $24 \mathrm{~h}$, increased ability to accumulated onto nanoHA1000 surface relatively to nanoHA830 surface. However, at $72 \mathrm{~h}$ this difference between biomaterials disappeared again and both presented similar bacterial numbers on their surface. For $\mathrm{Ci}=1.23 \times 10^{8}$ cells $/ \mathrm{mL}$ nanoHA1000 surface presented highest accumulation of S. epidermidis up to $48 \mathrm{~h}$ of incubation; however, these differences dis-appeared for $72 \mathrm{~h}$ of incubation (Figure 4(b)).

However, Ci used seems to have influenced bacterial attachment and accumulation on nanoHA surfaces over time (Figure $4(\mathrm{a})$ and (b)). At $24 \mathrm{~h}$, for $\mathrm{Ci}=1.23 \times 10^{5}$ cells $/ \mathrm{mL}$, the S. epidermidis amounts on both nanoHA surfaces were significantly lower when compared to 
those obtained for $\mathrm{Ci}=1.23 \times 10^{8}$ cells/mL. However, for longer periods ( 48 and $72 \mathrm{~h}$ ) the materials presented significant differences. For both $\mathrm{Ci}$ values nanoHA830 surface showed significant increase of bacterial accumulation for those time points, the adhesion being highest at $72 \mathrm{~h}$ for $\mathrm{Ci}=1.23 \times 10^{8}$ cells $/ \mathrm{mL}$ (Figure $4(\mathrm{a})$ and (b)). The nanoHA1000 surface did not present significant differences in bacterial accumulation between 48 and $72 \mathrm{~h}$, for both Ci values (Figure 4(a) and (b)).

$\mathrm{Ci}=1.23 \times 10^{8}$ cells $/ \mathrm{mL}$ was chosen to analyze the structure and morphology of biofilm formed on biomaterials surfaces over time. The SEM images showed different profiles of S. epidermidis attached and accumulated onto nanoHA830 and nanoHA1000 surfaces after 72 $\mathrm{h}$ of incubation (Figure 5), in accordance with the obtained results for biofilm density (Figure 5). After $24 \mathrm{~h}$, bacteria were spread, almost completely covering the nanoHA1000 surfaces (Figure 5). Later, the biofilm presented multilayered bacterial cells, embedded in EPS, which were already visible in the micrographs taken at $48 \mathrm{~h}$, and more intensively at $72 \mathrm{~h}$ (Figure 5). At this time point, that surface was completely covered by biofilm (Figure 5). Some cracks observed in almost all images may be attributed to biofilm shrinkage during the dehydration process. The nanoHA830 sur-face was never completely covered by the biofilm even after 72 $\mathrm{h}$ of incubation (Figure 5). The biofilm was composed of micro-colonies growing vertically like columns or stacks attached to the surface and there were no visible extracellular polymers connecting the bacteria (Figure 5).

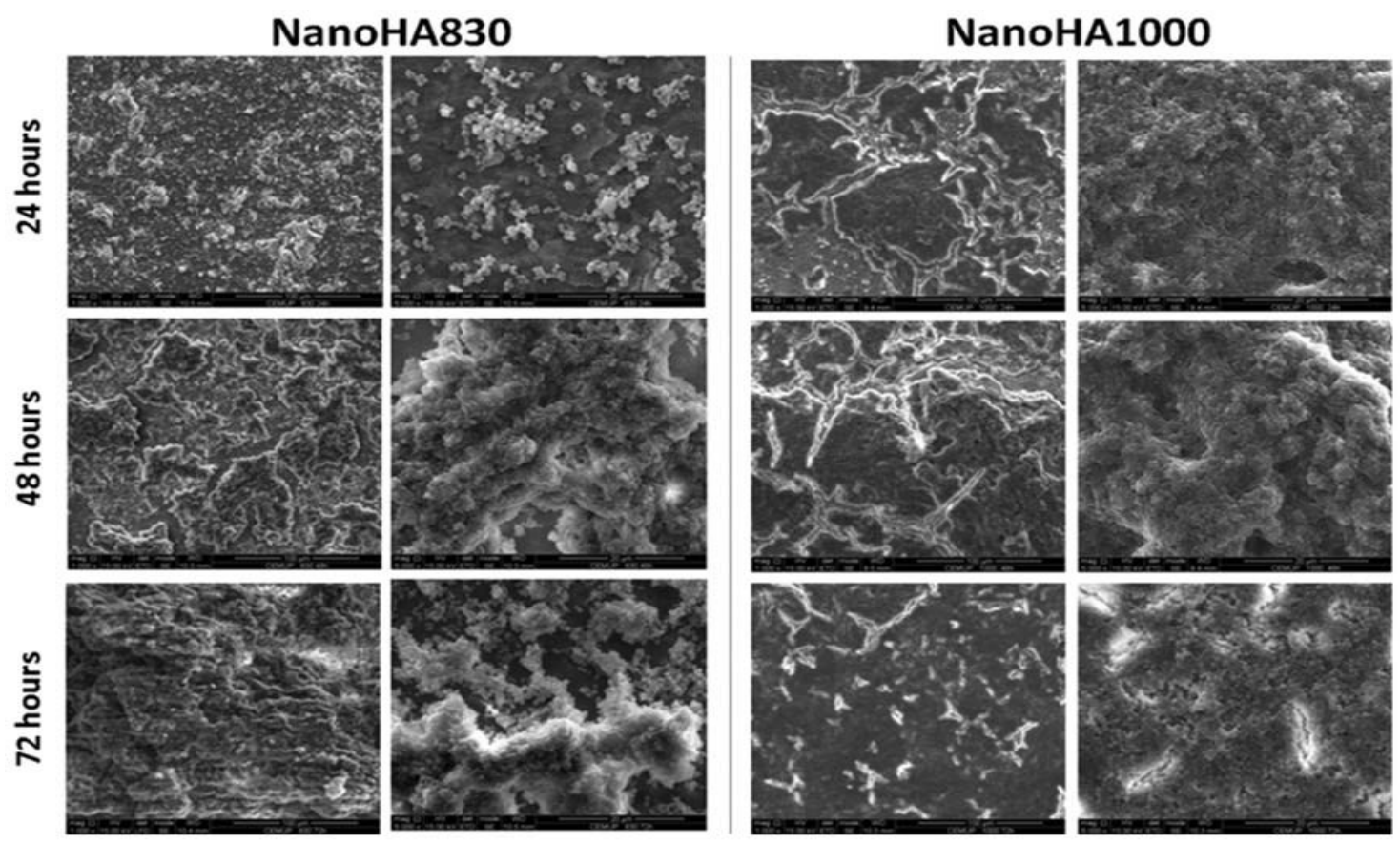

Figure 5. Images of biofilm growth on different biomaterials over time: nanoHA830 surface and nanoHAI 000 surface obtained by SEM using 1000 and 5000x magnification. 


\section{Discussion}

S. epidermidis is strongly associated with infections related to implants and medical devices. ${ }^{18,19}$ The development of infections in or around the indwelling bio- material devices, such as orthopedic prostheses, urinary tract and cardiovascular catheters, intraocular lenses and dentures, continues to be a key issue that hinders the use of such devices. While evidently detrimental to the healing process and overall wellbeing of the patient, bacterial attachment, while ensuring the formation of a biofilm, may also damage the performance of the device. ${ }^{20}$ NanoHA has been used as coating or bone substitute in orthopaedic devices, due to its properties such as biocompatibility, bioactivity, osteoconductivity and osteoinduction. ${ }^{2,21,22}$ However, information on the effect of nanoHA surface properties on bacterial adhesion and biofilm formation is scarce. ${ }^{20}$ In this work, the purpose was to study the influence of nanoHA surface characteristics in $S$. epidermidis RP62A biofilm formation. Therefore, two different initial inoculum concentrations $\left(\mathrm{C}_{\mathrm{i}}\right)$ were used in order to check if these $\mathrm{C}_{\mathrm{i}}$ would affect the biofilm formed on nanoHA surfaces differing exclusively on the effect of different sintering temperatures, while both keeping grain size within the nanoscale.

The results obtained showed different profiles for S. epidermidis attachment and accumulation onto nanoHA830 and nanoHA1000 surfaces. The nanoHA830 surface showed to be more resistant to S. epidermidis attachment an accumulation than the nanoHA1000 surface. Several factors may influence bacterial attachment and accumulation on a biomaterial surface, namely chemical composition, surface charge (zeta potential), hydrophobicity and $\mathrm{Ra}^{23-25}$

It is known that the sintering temperature affects the crystallographic characteristics and grain size of the ceramics. Previous results have indicated that sintering at these temperatures would increase crystallinity and present peaks typical of hydroxyapatite, as bTCP would be only present if sintering would occur above $1250^{\circ} \mathrm{C}^{26}$ The chemical characterization of nanoHA was performed by using Fourier transformed infrared spectroscopy (FT-IR) and both types of sintered nanoHA presented the same characteristics band for phosphate, hydroxyl groups and for $\mathrm{H}_{2} \mathrm{O}$ observed in the non-sintered nanoHA (data to be published). The SEM and AFM images showed that the grain size increased with increasing sintering temperature. Grain size growth depended, above all, on sintering temperature, which led to the mobility of pores located along grain boundaries that migrate at the same rate as the migrating grain boundaries, the material transport mechanism being surface diffusion. ${ }^{27,28}$

The hydrophilic or hydrophobic character of materials has an influence on bacterial adhesion. Most studies found a positive correlation between the substratum hydrophobicity and the bacteria adherence and accumulation. ${ }^{24,29}$ In our study, both nanoHA surfaces presented hydrophilic character, as described by others authors, ${ }^{30,31}$ although differences were observed in the number of bacteria accumulated onto nanoHA830 and nanoHA1000 surfaces, meaning that other properties beyond wettability might be influencing bacterial adhesion. Schwarz et al. ${ }^{32}$ showed that the hydrophilicity of titanium implant surface had no apparent effect on supragingival plaque biofilm formation. Several other studies suggested similar adhesions on hydrophilic and hydrophobic surfaces or even better adhesion have also been reported on hydrophilic substrata. ${ }^{33,34}$ Though there is still some controversy about the effect of wettability of the materials on bacterial adhesion and bio- film growth, factors such as surface free energy of the bacteria, the ionic strength of solution and the surface free energy, may influence bacterial adhesion. ${ }^{24,35-37}$ A higher or lower material surface free energy could either decrease or increase adhesion or make it either reversible or irreversible, depending on the experimental conditions. ${ }^{24}$

The material surface ZP is another parameter that may significantly influence bacterial adhesion, consequence of repulsive electrostatic interactions between bacteria and substrates surfaces. ${ }^{23,29}$ Montag et al. ${ }^{29}$ referred that the closer the ZP is to zero, the higher the accumulated bacteria density. Although bacterial susceptibility to positively charged surfaces has been reported, ${ }^{23}$ the relationship between bacterial adhesion and substratum surface charge remains unclear. Some kind of hydrophobic interactions between the cell and 
material surfaces apparently occurs, that may enable cells to overcome the repulsive forces active within a certain distance from the material surface, and attach irreversibly. ${ }^{35}$ On the other hand, some studies suggest that no correlation exists between bacterial adhesion and substratum charge. ${ }^{38}$ In our work, the obtained ZP values indicate that nanoHA presented a negative net charge in agreement with the charge shown by most ceramic materials, as described in the literature ${ }^{27,39-41}$; however, a correlation between surface ZP values and the amount of accumulated bacteria was not observed. Regarding material morphology, porous bioceramics configuration required for cell ingrowth offers highly irregular surface that may also allow bacterial colonization. ${ }^{39}$ In our study, however samples were prepared as 2 D surfaces and therefore porosity was not introduced, at least within the size range applicable for tissue regeneration. Still, the pore distribution for both biomaterials indicated the presence of some pores with diameters less than $600 \mathrm{~nm}$. This pore size is too small to allow the colonization of bacteria, which are about $1.0 \pm 0.13 \mu \mathrm{m}$. Kinnari et al. showed that HA did not have pores large enough to allow the internalization of Staphylococci, corroborating our results. By AFM and SEM analysis, it was also possible to observe that the aggregates size of nanoHA increased with increasing sintering temperature. Although these samples, due to their characteristics of "dense" materials, did not show very high porosities, these were still different and it was relevant to study whether these differences would affect biofilm formation.

The differences observed for $S$. epidermidis attachment and accumulation on different nanoHA surfaces may relapse on roughness and surface area of these bio- materials. The material Ra has been pointed out as cap- able of influencing bacterial adhesion, proliferation, biofilm formation and detachment of adherent bacteria to a given material. ${ }^{20,43,44}$ This is probably due to the fact that rough surfaces have greater surface areas ${ }^{35}$ and that depressions in the roughened surfaces provide more favorable sites for colonization, as crevices may protect bacteria from shear stresses. ${ }^{18,23}$ In our study, a correlation between roughness, surface area and amount of bacteria was observed, with nanoHA1000 displaying higher average roughness values and, simultaneously, larger amount of bacterial accumulation. Concerning $\mathrm{C}_{\mathrm{i}}$, it seems that this parameter has an influence on bacterial attachment and accumulation on nanoHA surfaces over time. For $1.23 \times 10^{5}$ cells $/ \mathrm{mL}$, the amounts of $S$. epidermidis on both nanoHA surfaces were significantly lower than those obtained for $1.23 \times 10^{8}$ cells $/ \mathrm{mL}$, after $24 \mathrm{~h}$ of incubation, which means that for shorter periods of culture, the choice of the initial inoculum concentration is relevant and may have an effect on the extent of in vitro adherence. However, for longer periods (48 and $72 \mathrm{~h}$ ), the nanoHA830 surface showed significant increase on bacteria accumulation for both $\mathrm{C}_{\mathrm{i}}$ values, the same not being observed for nanoHA1000 surface. The quasi- steady state equilibrium reached after $48 \mathrm{~h}$ on nanoHA1000 surface may be explained by bacterial co-adhesion. ${ }^{45}$ These results showed that the knowledge of the influence of inoculum is useful for further research, namely to assess bacterial adhesion to other biomaterials. ${ }^{46,47}$

The biofilm structure and morphology formed on these biomaterial surfaces, over time, were also assessed by SEM. A mature biofilm with EPS production and three-dimensional mushroom-like or pillar-like structures was observed, on both biomaterial surfaces. The SEM images support the quantitative results obtained, showing that $S$. epidermidis had more ability to attach and accumulate onto nanoHA1000 than onto nanoHA830 surface, over $72 \mathrm{~h}$ of incubation. NanoHA830 surfaces supported a different type of bio- film when compared to nanoHA1000 surface. This difference was noticed up to $72 \mathrm{~h}$, in terms of structure, EPS production and surface coverage. Even after $48 \mathrm{~h}$ of incubation, the nanoHA830 surface was never completely covered by the biofilm, which was composed of micro-colonies growing vertically like columns or stacks attached to the surface. Over this surface there were less or none extracellular polymers connecting the bacteria when compared to those observed on nanoHA1000 surface. This type of biofilm structures was already observed by other authors and was attributed to differences in shear stress and nutrients avail- ability. ${ }^{14,23,48}$ On nanoHA1000 surface, the bacteria were spread all over the surfaces, and after $48 \mathrm{~h}$ biofilms presented a compact structure composed of a multi- layered bacterial community, embedded into EPS. Similar structures were observed by SEM on S. epidermidis biofilms formed on central venous catheters. ${ }^{49}$ According to Patel et al. ${ }^{50}$ the EPS formed in S. epidermidis biofilm 
were commonly identified as the cause of biomaterial-centered infections. These EPS play a significant role in mediating the bacterial colonization of surfaces by facilitating cell adhesion to surfaces and internal biofilm cohesion, as well as, in the events following the initial steps of adhesion that include protection against phagocytosis, interference with the cellular immune response and reduction of antibiotic effects. ${ }^{9}$

The overall results indicate that not only the biomaterial surface characteristics have influence on S. epidermidis attachment and accumulation, but the initial inoculum also plays a role in this equation.

\section{Conclusions}

In this study, it was shown that both nanoHA surfaces presented hydrophilic character, but with differences in terms of ZP values, pore diameter, porosity, surface area, topography and roughness. The profiles of $S$. epidermidis attached and accumulated onto nanoHA830 and nanoHA1000 surfaces over $72 \mathrm{~h}$ of incubation were followed and the influence of $\mathrm{Ci}$ on biofilm formation on those nanoHA surfaces was studied. NanoHA830 surfaces showed to be more resistant to $S$. epidermidis attachment and accumulation than those of nanoHA1000. The biofilm formed on nanoHA830 presented differences in terms of structure, surface coverage and EPS production when compared to bio- film formed on nanoHA1000. The properties of biomaterials that influence more S. epidermidis attachment and accumulation were Ra and actual surface area that showed a correlation with bacterial density. Others properties of biomaterials namely wettability, ZP and porosity apparently did not show a strong effect on $S$. epidermidis biofilm formation. $\mathrm{C}_{\mathrm{i}}$ influenced bacterial attachment and accumulation on nanoHA surfaces over time. The choice of the initial inoculum concentration seems to be relevant as it may have an effect on the extent of adherence and this will be a critical aspect for human health if these materials are used in implantable devices. The biomaterial surface characteristics determine the rate of microbial attachment to substrata and consequently are related to biofilm- associated infections on biomaterials.

The knowledge of the influence of inoculum concentration is useful for research purposes, namely to assess bacterial adhesion to biomaterials, aiming at preventing or reducing infections and to choose the most appropriate antibiotic therapy, as the inoculum concentration may alter antibiotic activity.

\section{Acknowledgements}

The provision of nanoHA (nanoXIM) by FLUIDINOVA, (Maia-Portugal) is greatly acknowledged. Also, the support of Dr Miguel Angel Rodriguez from ICV (CSIC- Madrid) in the linear expansion behavior characterization of nanohydroxyapatite is acknowledged with gratitude.

\section{Funding}

J.B. is supported by a research grant from FEDER funds through COMPETE and by FCT Fundação para a Ciência e a Tecnologia in the framework of the project NanoBiofilm (PTDC/SAU-BMA/111233/2009).

\section{Conflicts of interest}

The authors confirm that there are no known conflicts of interest associated with this publication and there has been no significant financial support for this work that could have influenced its outcome. 


\section{References}

1. Singh C, Hu Y, Khanal BP, et al. Striped nanowires and nanorods from mixed. Nanoscale 2011; 3: 3244-3250.

2. Deligianni DD, Katsala ND, Koutsoukos PG, et al. Effect of surface roughness of hydroxyapatite on human bone marrow cell adhesion, proliferation, differentiation and detachment strength. Biomaterials 2001; 22: 87-96.

3. Ferraz MP, Monteiro FJ and Manuel CM. Hydroxyapatite nanoparticles: a review of preparation methodologies. J Appl Biomater Biomech 2004; 2: 74-80.

4. Ferraz MP, Mateus AY, Sousa JC, et al. Nanohydroxyapatite microspheres as delivery system for antibiotics: release kinetics, antimicrobial activity, and interaction with osteoblasts. J Biomed Mater Res A 2007; 81: 994-1004.

5. Furuzono T, Walsh D, Sato K, et al. Effect of reaction temperature on the morphology and size of hydroxyapa- tite nanoparticles in an emulsion system. J Mater Sci Lett 2001; 20: 111114.

6. Mateus AYP, Barrias CC, Ribeiro C, et al. Comparative study of nanohydroxyapatite microspheres for medical applications. J Biomed Mater Res Part A 2008; 86A: 483-493.

7. Vallet-Regi M. Ceramics for medical applications.

J Chem Soc Dalton Trans 2001; 2: 97-108.

8. Rimondini L, Fini M and Giardino R. The microbial infection of biomaterials: a challenge for clinicians and researchers. A short review. J Appl Biomater Biomech 2005; 3:1-10.

9. McCann MT, Gilmore BF and Gorman SP. Staphylococcus epidermidis devicerelated infections: pathogenesis and clinical management. $J$ Pharm Pharmacol 2008; 60: 1551-1571.

10. O'Gara JP and Humphreys H. Staphylococcus epidermi- dis biofilms: importance and implications. J Med Microbiol 2001; 50: 582-587.

11. Yao YF, Sturdevant DE, Villaruz A, et al. Factors char- acterizing staphylococcus epidermidis invasiveness deter- mined by comparative genomics. Infect Immun 2005; 73: 1856-1860.

12. Cerca N, Pier GB, Vilanova $M$, et al. Influence of batch or fed-batch growth on Staphylococcus epidermidis biofilm formation. Lett Appl Microbiol 2004; 39: 420-424.

13. Melo LF and Bott TR. Biofouling in water systems.

Exp Therm Fluid Sci 1997; 14: 375-381.

14. Monds RD and O'Toole GA. The developmental model of microbial biofilms: ten years of a paradigm up for review. Trends Microbiol 2009; 17: 73-87.

15. Wang IW, Anderson JM and Marchant RE. Staphylococcus-epidermidis adhesion to hydrophobic biomedical polymer is mediated by platelets. $J$ Infect Dis 1993;167:329-336.

16. Gadelmawla ES, Koura MM, Maksoud TMA, et al. Roughness parameters. J Mater Process Technol 2002; 123: 133-145.

17. Ho TA, Papavassiliou DV, Lee LL, et al. Liquid water can slip on a hydrophilic surface. Proc Natl Acad Sci 2011; 108:16170-16175.

18. Sousa $C$, Teixeira $P$ and Oliveira R. Influence of surface properties on the adhesion of staphylococcus epidermidis to acrylic and silicone. Int J Biomater 2009; ID 718017.

19. Fonseca AP, Granja PL, Nogueira JA, et al. Staphylococcus epidermidis RP62A adhesion to chem- ically modified cellulose derivatives. J Mater Sci: Mater Med 2001; 12: 543-548.

20. Bazaka K, Jacob MV, Crawford RJ, et al. Efficient sur- face modification of biomaterial to prevent biofilm for- mation and the attachment of microorganisms. Appl Microbiol Biotechnol 2012; 95: 299-311.

21. Tampieri A, Celotti G, Sprio S, et al. Porosity-graded hydroxyapatite ceramics to replace natural bone. Biomaterials 2001; 22: 1365-1370.

22. Fenoglio I, Fubini B, Ghibaudi EM, et al. Multiple aspects of the interaction of biomacromolecules with inorganic surfaces. Adv Drug Delivery Rev 2011; 63: 1186-1209. 
23. Katsikogianni M and Missirlis YF. Concise review of mechanisms of bacterial adhesion to biomaterials and of techniques used in estimating bacteria-material inter- actions. Eur Cell Mater 2004; 8: 37-57.

24. Pereni CI, Zhao Q, Liu Y, et al. Surface free energy effect on bacterial retention. Colloids Surf B: Biointerfaces 2006; 48: 143-147.

25. Clauss M, Trampuz A, Borens 0 , et al. Biofilm formation on bone grafts and bone graft substitutes: comparison of different materials by a standard in vitro test and microcalorimetry. Acta Biomater 2010; 6: 3791-3797.

26. Laranjeira MS, Fernandes and Monteiro FJ. Innovative macroporous granules of nanostructured hydroxyapatite agglomerates: bioactivity and osteoblast-like cell behaviour. J Biomed Mater Res A 2010; 95A: 891-900.

27. Muralithran $\mathrm{G}$ and Ramesh $\mathrm{S}$. The effects of sintering temperature on the properties of hydroxyapatite. Ceram Int 2010; 26: 221-230.

28. He ZM, Ma J and Wang C. Constitutive modeling of the densification and the grain growth of hydroxyapatite cer- amics. Biomaterials 2005; 26: 1613-1621.

29. Montag D, Frant M, Horn H, et al. Dependence of the initial adhesion of biofilm forming Pseudomonas putida mt2 on physico-chemical material properties. Biofouling 2012; 28: 315327.

30. Vandiver J, Dean D, Patel N, et al. Nanoscale variation in surface charge of synthetic hydroxyapatite detected by chemically and spatially specific high-resolution force spectroscopy. Biomaterials 2005; 26: 271-283.

31. Tihan TG, Ionita MD, Popescu RG, et al. Effect of hydrophilic- hydrophobic balance on biocompatibility of poly(methyl methacrylate) (PMMA)- hydroxyapatite (HA) composites. Mater Chem Phys 2009; 118: 265-269.

32. Schwarz F, Sculean A, Wieland M, et al. Effects of hydrophilicity and microtopography of titanium implant surfaces on initial supragingival plaque biofilm forma- tion. A pilot study. Mund Kiefer GesichtsChir 2007; 11: 333-338.

33. Quirynen M, Dierickx K and Steenberghe D. Effects of surface roughness and free energy on oral bacterialadhe- sion. In: An Y and Friedman R (eds) Handbook of bac-terial adhesion - Principles, Methods, and Applications, Part I. Towota, USA: Humana Press, 2000, pp.91-102.

34. Anselme K, Ploux L and Ponche A. Cell/material inter- faces: influence of surface chemistry and surface topog- raphy on cell adhesion. J Adhes Sci Technol 2010; 24: 831852.

35. Donlan RM. Biofilms: microbial life on surfaces. Emerg Infect Dis 2002; 8: 881-890.

36. Cerca N, Pier GB, Vilanova M, et al. Quantitative ana- lysis of adhesion and biofilm formation on hydrophilic and hydrophobic surfaces of clinical isolates of staphylo- coccus epidermidis. Res Microbiol 2005; 156: 506-514.

37. Pereira MA, Alves MM, Azeredo J, et al. Influence of physico-chemical properties of porous microcarriers on the adhesion of an anaerobic consortium. $J$ Ind Microbiol Biotechnol 2000; 24: 181-186.

38. Li B and Logan BE. Bacterial adhesion to glass and metal-oxide surfaces. Colloids Surf B Biointerfaces 2004; 36: 81-90.

39. Lopes MA, Monteiro FJ, Santos JD, et al. Hydrophobicity, surface tension, and zeta potential measurements of glass-reinforced hydroxyapatite com- posites. J Biomed Mater Res 1999; 45: 370-375.

40. Suzuki T, Ohashi R, Yokogawa Y, et al. Initial anchoring and proliferation of fibroblast L-929 cells on unstable surface of calcium phosphate ceramics. $J$ Biosci Bioeng 1999; 87: 320-327.

41. Ferraz MP, Monteiro FJ, Serro AP, et al. Effect of chem- ical composition on hydrophobicity and zeta potential of plasma sprayed HA/CaO P2O5 glass coatings. Biomaterials 2001; 22: 3105-3112.

42. Kinnari TJ, Esteban J, Martin-de-Hijas NZ, et al. Influence of surface porosity and 
pH on bacterial adher- ence to hydroxyapatite and biphasic calcium phosphate bioceramics. J Med Microbiol 2009; 58: 132-137.

43. Chin MY, Sandham A, De Vries J, et al. Biofilm forma- tion on surface characterized micro-implants for skeletal anchorage in orthodontics. Biomaterials 2007; 28: 2032-2040.

44. Carlen A, Nikdel K, Wennerberg A, et al. Surface char- acteristics and in vitro biofilm formation on glass iono- mer and composite resin. Biomaterials 2001; 22: 481-487.

45. Kolenbrander PE, Andersen RN, Kazmerzak KM, et al. Coaggregation and coadhesion in oral biofilms. Community structure and co operation in biofilms. SYMPOSIA Society for General Microbiology. Cambridge, UK: Cambridge University Press, 2000.

46. Mizunaga S, Kamiyama T, Fukuda Y, et al. Influence of inoculum size of Staphylococcus aureus and Pseudomonas aeruginosa on in vitro activities and in vivo efficacy of fluoroquinolones and carbapenems. J Antimicrob Chemother 2005; 56: 91-96.

47. Sanders DL, Kingsnorth AN, Lambie J, et al. An experi- mental study exploring the relationship between the size of bacterial inoculums and bacterial adherence to pros- thetic mesh. Surg Endosc 2013; 27: 978-985.

48. Katsikogianni MG and Missirlis YF. Interactions of bac- teria with specific biomaterial surface chemistries under flow conditions. Acta Biomater 2010; 6: 1107-1118.

49. Rohde H, Frankenberger S, Zahringer U, et al. Structure, function and contribution of polysaccharide intercellular adhesin (PIA) to Staphylococcus epidermidis biofilm formation and pathogenesis of biomaterial-associated infec- tions. Eur J Cell Biol 2010; 89: 103-111.

50. Patel JD, Ebert M, Ward R, et al. S. epidermidis bio- film formation: effects of biomaterial surface chemistry and serum proteins. J Biomed Mater Res A 2007; 80: $742-751$. 